\title{
A New Plane of Elevation: The Superficial Fascial Plane for Perforator Flap Elevation
}

\author{
Joon Pio Hong, MD, PhD, MMM, ${ }^{1}$ Dong Hoon Choi, MD ${ }^{1}$ Hyunsuk Suh, MD ${ }^{2}$ \\ Dewi Aisiyah Mukarramah, MD ${ }^{1}$ Talal Tashti, MD ${ }^{1}$ Kyungjin Lee, $M^{1}$ Chiseoun Yoon, MD $^{3}$ \\ Address for correspondence Joon Pio Hong, MD, PhD, MMM, \\ Department of Plastic Surgery, Asan Medical Center, University of \\ Ulsan, 388-1 Poongnapdong Songpagu, Seoul, Korea 138-736 \\ (e-mail: joonphong@amc.seoul.kr).
}

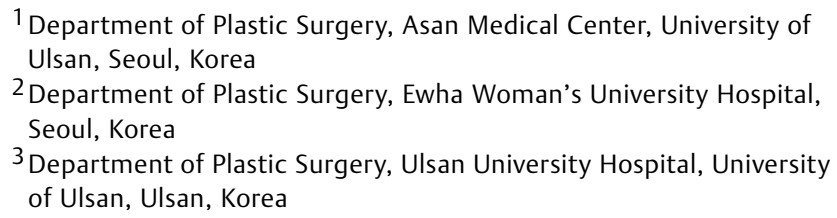

J Reconstr Microsurg 2014;30:491-496.

\begin{abstract}
Background The aim for microsurgical reconstruction has broadened from achieving functional to also providing good esthetic outcomes. The perforator flaps are widely used for this goal. However, perforator flaps can still be bulky especially to resurface the skin defect. We hypothesized that elevation from the superficial fascial plane can obtain a thin and viable flap.

Method In this retrospective study, we report consecutive perforator flaps elevated at the superficial fascial plane from November 2007 to July 2013. Total of 304 flaps which were 196 superficial circumflex iliac perforator (SCIP) flaps, 81 anterolateral thigh (ALT) flaps, and 27 gluteal artery perforator (GAP) flaps were reviewed.

Result The patient group composed of 189 male and 115 female patients with an average age of 35.8 years. The average body mass index was $23.5 \mathrm{~kg} / \mathrm{m}^{2}$ (range, 15.91 $34.57 \mathrm{~kg} / \mathrm{m}^{2}$ ). All 304 flaps were successfully elevated with this approach with at least one viable perforator. The thickness averaged approximately $6 \mathrm{~mm}$ for ALT flap (range, 4-11 mm), $5 \mathrm{~mm}$ for SCIP flap (range, 3-12 mm), and $8.5 \mathrm{~mm}$ for GAP flap (range, 5-11 $\mathrm{mm}$ ). Complete survival was noted in 282 flaps, partial loss of flaps requiring secondary

\section{Keywords}

- superficial fascia

- flap elevation

- SCIP

- ALT

- GAP procedures in 6 cases, partial loss healing secondarily in 8 cases, and total loss in 9 cases. During the average follow-up of 34 months, secondary debulking procedures were required in six flaps.

Conclusion This new approach of elevation on the superficial fascia is reliable, provides a viable tissue, and is able to obtain a thin flap achieving good functional and esthetic outcome.
\end{abstract}

The goals of microsurgery reconstruction are functional outcome and esthetic result. To achieve these goals, we often need a thin flap which is pliable and viable. Perforator flaps have been extensively used for these purposes from head and neck reconstruction, trunk reconstruction, and extremity reconstruction. But often when reconstructing the extremity or the foot, the bulk of the perforator flaps makes it difficult to achieve good contour at a single stage. ${ }^{1}$

received

November 10, 2013

accepted after revision

December 1, 2013

published online

February 19, 2014

Among the perforator flaps, one of the most used perforator flaps is the anterolateral thigh (ALT) flap. Initially described by Baek and Song et al and with refinements from Wei et al, it has become one of the ideal flaps for reconstruction providing reliable anatomy, long pedicles, thin flaps, sensation, and a reasonable donor site scar with minimal morbidity. ${ }^{2-4}$ The current method of elevation of the flap is either subfascial where the flap is elevated with the deep muscle fascia or

Copyright $\odot 2014$ by Thieme Medical Publishers, Inc., 333 Seventh Avenue, New York, NY 10001, USA. Tel: +1(212) 584-4662.
DOI http://dx.doi.org/ 10.1055/s-0034-1369807. ISSN 0743-684X. 
suprafascial where the elevation is made just above the deep fascial plane. ${ }^{4}$ In either case, the flap frequently remains too bulky to resurface the defect. Immediate debulking procedures can follow but may result in partial loss of the flap. ${ }^{5}$ Microdissection can be applied with better success to achieve a thin flap but may be too tedious and time consuming. ${ }^{6}$ Thus, secondary debulking procedures are chosen to minimize the flap loss and procedures such as liposuction or surgical resection follows to achieve better contour of the reconstruction site. But if possible, one can aim to have the ideal reconstruction at a given single stage such as the concept of the reconstructive elevator. ${ }^{7}$

To achieve a thin flap, minimize debulking procedures, improve donor contour, and establish a reliable plane of harvest, we hypothesized that elevation on the superficial fascia would deliver these goals. In this article, we reviewed the feasibility and efficacy of this applications for the ALT perforator, superficial circumflex iliac perforator (SCIP), and gluteal artery perforator (GAP) flaps.

\section{Patients and Method}

This a retrospective study approved by the institutional review board of Asan Medical Center. We reviewed consecutive perforator flaps being elevated at the superficial fascial plane from November 2007 to July 2013. Total of 304 flaps were reviewed. Three types of perforator flaps were used in this approach: 196 SCIP flaps, 81 ALT perforator flaps, and 27 GAP flaps. The flaps were used to reconstruct after cancer resection in 96 cases, diabetic foot in 80 cases, trauma in 83 cases (acute 5 , subacute 21 , and chronic with osteomyelitis in 57cases), posttrauma soft tissue contractures in 5 cases, vascular malformation in 12 cases, and other causes in 29 cases.

\section{Surgical Technique}

Preoperative multidetector row computed tomography angiograms or conventional angiograms when hardware was used were obtained in the patients to evaluate the vascular status of the extremity. It can help to identify the perforators rising from the thigh, gluteal, and groin region. Preoperative markings of perforators are made based on the anatomical landmarks as described by Wei et al for ALT flaps, Hong et al for SCIP flaps, and Ahmadzadeh et al for GAP flaps. ${ }^{8-10}$ In addition, a hand held Doppler was used to locate the actual perforator for a free style approach.

After complete excision or debridement, the flap dimension is measured, recipient vessels isolated, and the donor pedicle length is estimated. When using a perforator as recipient, a strong visible pulse of the perforator is the main indicator for use. ${ }^{11}$ For flaps especially GAP and SCIP, a pinch test of the skin should be made to ensure primary closure of the donor site. In large defects, ALT flaps are preferred and sometimes may need donor closure with skin grafts.

The elevation is a free style approach under loupe magnification and begins from the either lateral or medial border of the flap. The incision is made deep to the superficial fascia dividing superficial and deep fat (-Fig. 1A). The superficial fascia is between the large deep fat lobules and smaller superficial fat lobules. The small lobules suddenly become larger as it passes a very thin fascia-like structure ( $\mathbf{- F i g}$. 1B). This may be more apparent in patients with high body mass index (BMI). It is easier to identify this thin white film-like fascia while retracting the skin from both sides of the incision. After locating the fascia, then elevation is made on this plane until the perforator marked previously on the skin is reached. Then, the same approach is made from the contralateral side. There can be multiple perforators identified along this plane. On the basis of the pulse and diameter of the vessels, a single perforator is chosen and
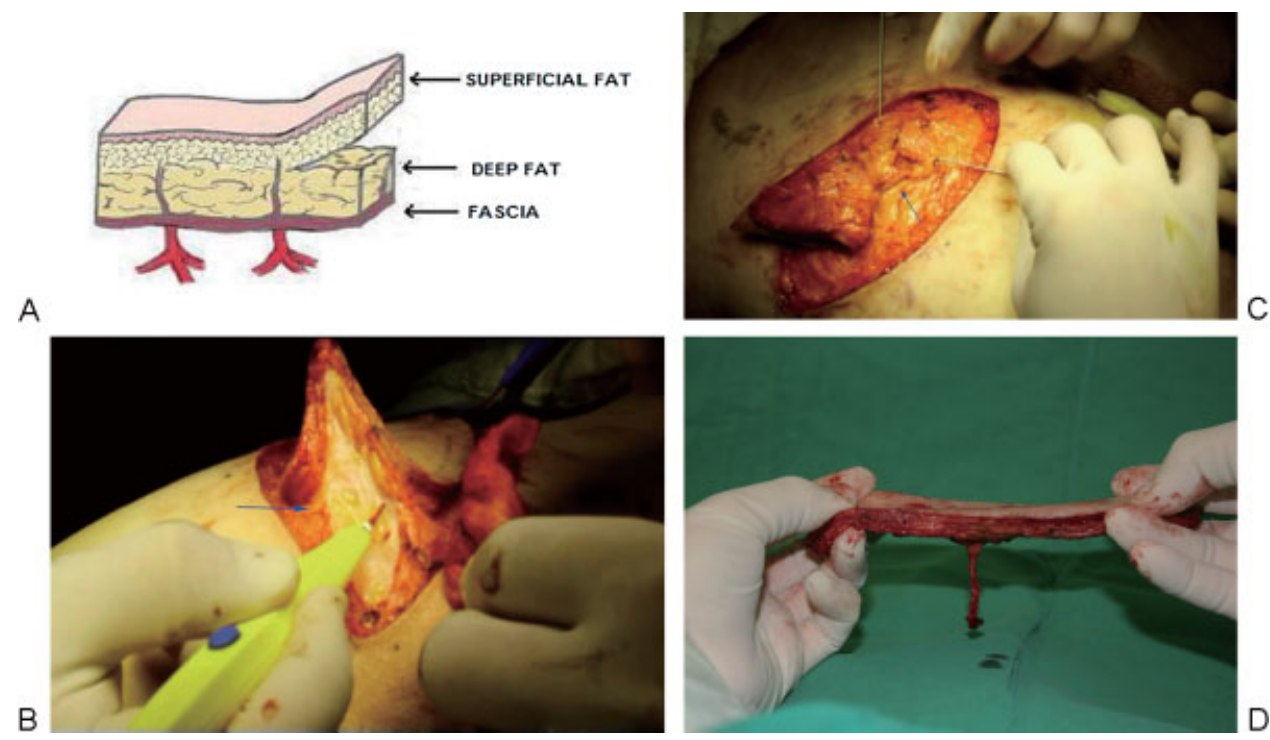

Fig. 1 (A) Exhibiting the superficial fascial plane between the deep and superficial fat. (B) Note that there is a white film-like plane (arrow) during the elevation of a gluteal artery perforator (GAP) flap. (C) Once the pedicle is identified (arrow), it can be dissected proximally toward the source vessel. (D) The thin GAP flap after elevation. 


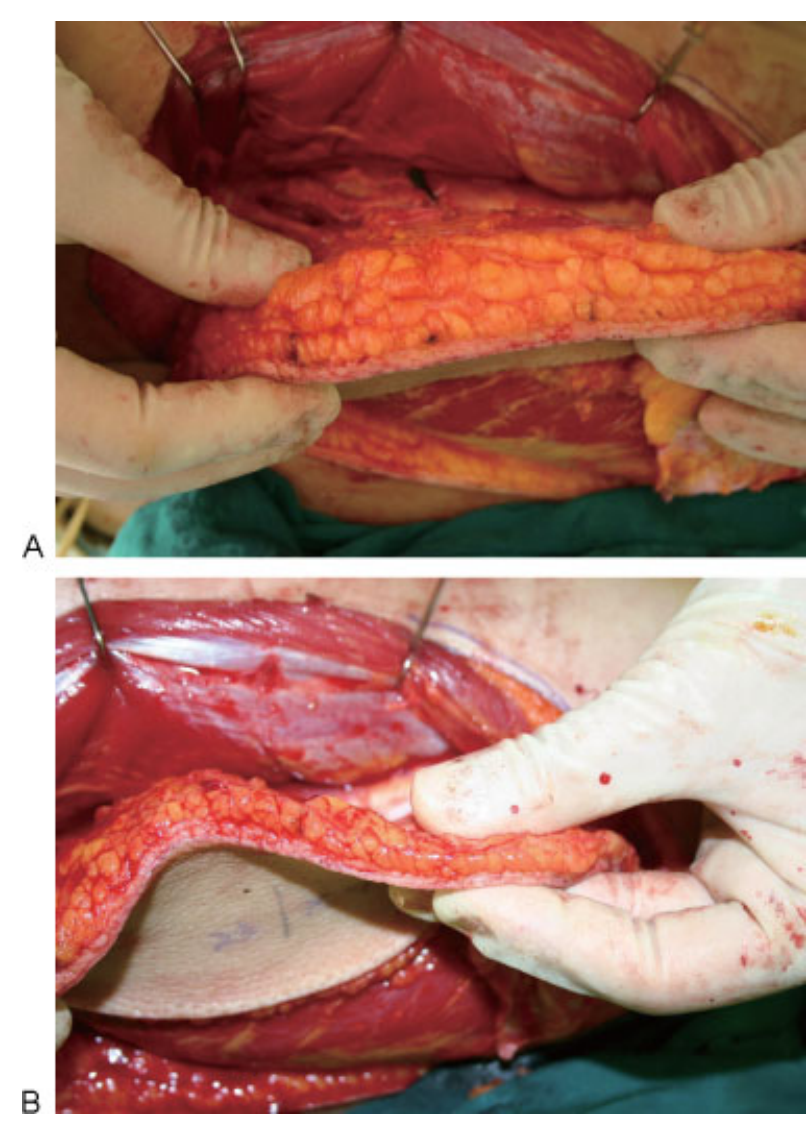

Fig. 2 (A) An anterolateral thigh (ALT) perforator flap is elevated above the deep fascia. (B) Demonstrating the reduced thickness once the deep fat is removed from the ALT perforator flap.

dissected into the deep fascia and muscle (-Fig. 1C). The fat around the perforator can be skeletonized or maintained with a cuff of fat. We prefer to skeletonize the pedicel as it allows identifying relevant branches to the flap. After reaching adequate length the pedicle is cut and made ready for microsurgery (-Fig. 1D). - Fig. 2 demonstrates an example of reduced thickness once the deep fat is removed from the ALT perforator flap.

\section{Results}

Between November 2007 and February 2013, the patient group was composed of 189 male and 115 female patients with an average age of 35.8 years (range, $6-77$ years). The average BMI was $23.5 \mathrm{~kg} / \mathrm{m}^{2}$ (range, $15.91-34.57 \mathrm{~kg} / \mathrm{m}^{2}$ ). All 304 flaps were successfully elevated on at least a single viable perforator with this approach. - Table 1 summarizes the results.

Total of 81 cases of anterolateral thigh free flaps were elevated on the superficial fascia. Primary closure of the donor site was performed in 18 cases (average flap size, $107.5 \mathrm{~cm}^{2}$; range, $\left.4 \times 7-25 \times 8 \mathrm{~cm}\right)$. Skin graft with acellular allogeneic dermal matrix (CGderm, CGBio Corporation, Korea) was required in rest of the cases, as the flap was too large and the donor site was not able to be closed primarily (average flap size, $175.1 \mathrm{~cm}^{2}$; range, $10 \times 7-30 \times 18 \mathrm{~cm}$ ). All 81 flaps were successfully elevated with at least one viable perforator with this approach. The average flap thickness was $6 \mathrm{~mm}$ (range, 4-8 mm). Total flap loss was noted in two cases, resulting in a $98 \%$ survival rate. Five flaps had partial loss, two flaps needed secondary procedures, and three flaps healed with secondary intention.

The elevation on the superficial fascia was performed for superficial circumflex iliac artery perforator free flap in 196 cases. The average size of the SCIP flap was $75.5 \mathrm{~cm}^{2}$ (range, $5 \times 3.5-25 \times 7 \mathrm{~cm}$ ). The average length of the pedicle was $5 \mathrm{~cm}$ with range from 2.5 to $7 \mathrm{~cm}$. The average diameter of the artery was $0.7 \mathrm{~mm}$ with range of 0.4 to $1.2 \mathrm{~mm}$. In 73 cases, only 1 vein was used, superficial vein was used in 32 cases overall. Average thickness of the flap was $5 \mathrm{~mm}$ (range, 3-12 mm). All donor sites were closed primarily. With the exception of seven flap complete losses, 97\% of the flaps survived. Partial loss was noted in six flaps, where five flaps needed secondary procedures such as skin grafts and one flap healed without further surgery. - Fig. 3 shows a 41-year-old female patient with sarcoma of the first toe reconstructed with an SCIP flap elevated on a superficial fascia. The follow-up at 6 months shows good contour of the toe compared with the normal first toe.

A total of 27 superior gluteal artery perforator flaps were successfully elevated on a single viable perforator on the superficial fascia plane. The average size of the GAP flap was $125 \mathrm{~cm}^{2}$ (range, $9 \times 4-25 \times 12 \mathrm{~cm}$ ). The average length of the pedicle was $5.5 \mathrm{~cm}$ (range, $3-8 \mathrm{~cm}$ ). The average diameter of the artery was $0.65 \mathrm{~mm}$ (range, $0.4-1.3 \mathrm{~mm}$ ). There were two accompanying veins for the GAP perforator in all the flaps but six flaps drained into single vein. The average thickness of the flap was $8.5 \mathrm{~mm}$ (range, 5-11 mm). All flaps survived completely with exception of two cases which had partial loss but eventually healed secondarily. Primary closure of the donor site was performed in all the patients.

During the average follow-up of 34 months (range, 3-69 months). During this time, secondary debulking procedures

Table 1 Summary of the flaps harvested on the superficial fascial plane

\begin{tabular}{|l|l|l|l|l|l|l|l|}
\hline & No. of cases & Thickness $(\mathbf{m m})$ & BMI $\left(\mathbf{k g} / \mathbf{m}^{2}\right)$ & Total loss & $\begin{array}{l}\text { Partial loss with } \\
\text { 2 degrees intension }\end{array}$ & $\begin{array}{l}\text { Partial loss with } \\
\text { 2 degrees healing }\end{array}$ & $\begin{array}{l}\text { 2 degrees } \\
\text { debulking }\end{array}$ \\
\hline ALT & 81 & 6 & 23.6 & 2 & 2 & 3 & 3 \\
\hline SCIP & 196 & 5 & 23.4 & 7 & 1 & 5 & 3 \\
\hline GAP & 27 & 8.5 & 23.9 & 0 & 2 & 0 & 0 \\
\hline Total & 304 & & & 9 & 5 & 8 & 6 \\
\hline
\end{tabular}

Abbreviations: ALT, anterolateral thigh; BMI, body mass index; GAP, gluteal artery perforator; SCIP, superficial circumflex iliac perforator. 

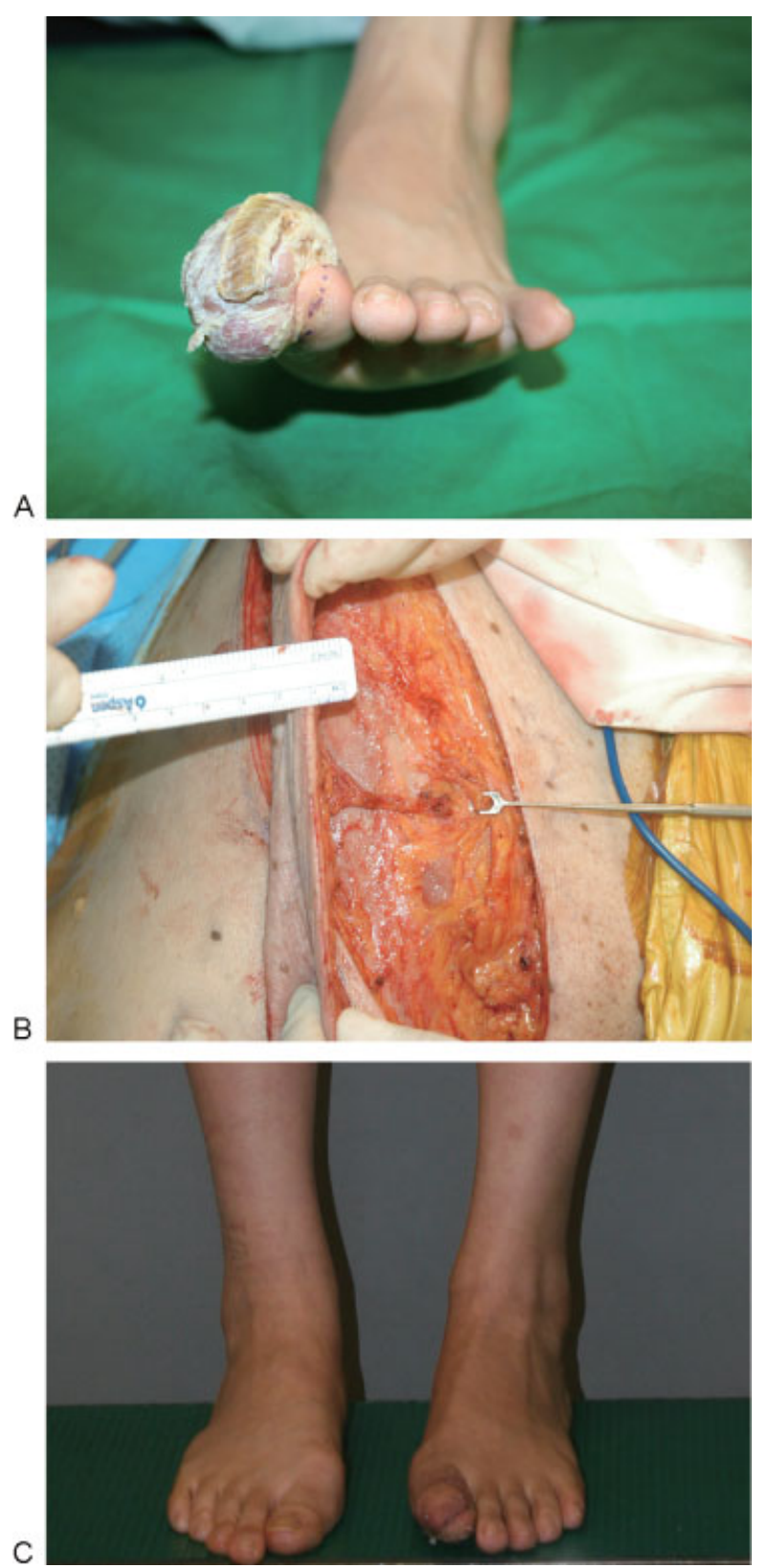

Fig. 3 (A) A 41-year-old female patient with sarcoma of the first toe is noted. (B) After wide excision, the defect is reconstructed with a superficial circumflex iliac perforator flap elevated on a superficial fascia. (C) The follow-up at 6 months shows good contour of the toe compared with the normal first toe.

were required in six flaps. In the three ALT flaps, patients had BMI over $29 \mathrm{~kg} / \mathrm{m}^{2}$ and in three SCIP flaps the BMI was over 26 $\mathrm{kg} / \mathrm{m}^{2}$. The GAP flap cases did not need debulking procedures. Among the 62 cases of plantar foot reconstruction, there was no ulceration after reconstruction in 39 of the sensory intact patients, but 4 cases of recurred ulceration was noted among the 23 patients without sensation ( - Table $\mathbf{1}$ ).

\section{Discussion}

Using the superficial fascial plane in flap elevation is a novel approach in resurfacing the defect. Until now, two previous described layers for elevating perforator flaps were either subfascial or the suprafascial planes. Subfascial approach is made by incising the deep fascia, and then elevating beneath this fascia which makes it easier to identify the perforators before it penetrates the fascia. It is easier and may be faster to elevate the flap., ${ }^{4,9,12}$ However, this approach leaves an unwanted donor site morbidity such as muscle hernia and unsightly scar if the donor site is not closed primarily. Also, the deep fascia itself adds extra thickness to the flap making it bulky for some reconstruction. The suprafascial approach elevates the flap above the fascia until the perforator is reached and then opening the hiatus of the deep fascia and dissecting the perforator proximally toward the source vessel. Although the risk for muscle hernia is reduced by keeping the deep fascia intact, the risk for skin graft loss and depression of scar remains a problem if the donor site is not closed primarily. Because of the deep layer fat, it may not be adequate for resurfacing defects with thin surrounding skin. In both approaches, immediate intraoperative or secondary debulking of the flap still can be required to obtain a natural donor site contour. However, during the immediate intraoperative debulking, the risk for partial necrosis may increase as the debulking procedures are performed without a distinction of an anatomical plane. ${ }^{13,14}$ The best way may be to dissect with direct visualization of the small branches traveling into the dermal plexus under a microscope. ${ }^{6}$ But this maybe a very tedious and a time consuming task.

We hypothesized that the superficial plane could be a reliable elevation plane providing safety, viability, and a thin flap. The knowledge of the superficial fascia and the plane between deep and superficial fat has opened a safe way to debulk a flap to a reasonable thickness. ${ }^{5,15,16}$ So by using this plane, we can obtain a thin flap without the need for additional debulking after the elevation. Although anatomical reports have stressed the importance of the vascular communication between the deep fascia and the subdermal plexus, the role of perfusion in these communication has not been evaluated. ${ }^{17}$ According to the perforasome concept by Saint-Cyr et al, viability of flap with superficial fascia plane will be derived from perfusion of the perforator and the ability of indirect linking vessels connecting to each other. ${ }^{18,19}$ On the basis of this knowledge, a large thin flap will survive even though without direct linking vessels. ${ }^{13,17}$ Therefore, despite debulking the deep fat where the direct linking vessels might be found, the thin flap harvested on the superficial plane will still have adequate blood supply. This hypothesis was proven to be an adequate one as 304 consecutive flaps elevated on the superficial fascia had a $97 \%$ survival rate and the largest dimension reached up to $30 \times 18 \mathrm{~cm}$ based on a single perforator.

In our series, we evaluated the most common perforator flaps used in our practice. The ALT perforator, SCIP, and the GAP flaps are the workhorses based on the required size of the flap, position of the patient during surgery, and the morbidity of the donor site. Basically, ALT perforator flaps are chosen for large or defects that needed a chimeric form of flap including muscles. When the defect was smaller and required only a short pedicle, SCIP was the choice. For a moderate size wounds on the posterior 
aspect of the leg, we chose the GAP. In all these cases, they had a superficial fascial plane. In patients with low BMI, the deep fat layer was less evident but had some deep fat underneath the superficial fascia. When harvesting the ALT, the superficial fascia becomes more evident as the harvest moves cephalic toward the upper thigh. Also, the superficial nerve becomes a good landmark to realize that you are in the right plane, as the nerve is always situated in the deep fat layer. For SCIP flap, it is crucial to stay above the deep fat plane. As the lymph nodes are situated in the deep fat layer, injury to the lymph node can increase the donor site morbidity by causing prolong lymphorrhea. The GAP flap has the most distinctive superficial fat plane. ${ }^{20}$ Even in the low BMI patients, there is always a thick layer of deep and superficial fat thus making the superficial fascial plane evident.

An interesting observation that we have found is that superficial fat layer and the thickness of the skin is different among the flaps. The flap was thinnest in the SCIP flap with an average of $5 \mathrm{~mm}$ and thickest in the GAP flap with average of $8.5 \mathrm{~mm}$. This may be due to the character of human fat deposition throughout our body and the thickness of the skin itself. ${ }^{20,21}$ But despite the thinness of these flaps, debulking was still required in three ALT and three SCIP cases. This is most likely due to the fact that these flaps were used to resurface the defect requiring a very thin flap. In most of the reconstruction involving the GAP flaps were posterior aspect of heals or calves which needed a relatively thick flap. Nevertheless, the debulking was required in six patients with relatively high BMIs indicating that the superficial fat layer can be thick in patients with high BMIs and may require additional debulking. But with far less bulk, even in obese patients, this approach can provide an easier closure of the defect and a simpler secondary debulking procedure.

Disadvantages for this approach can be the learning curve required to identify the superficial fascial plane, the ability to dissect and isolate perforators, and the possibility of a short pedicle. But once comfortable elevating on this plane, one can see the small vessels actually branching from the perforator and include it on the flap under loupe magnification.

In conclusion, superficial fascia is a new approach for elevating flaps. It has advantages such as providing a thin flap allowing increased versatility to achieve better contour of flap, minimizing the need for secondary debulking, and being hemodynamically reliable.

\section{Conflict of Interest}

None of the author has any conflict of interest regarding any materials mentioned in this article.

\section{References}

1 Pribaz JJ, Chan RK. Where do perforator flaps fit in our armamentarium? Clin Plast Surg 2010;37(4):571-579, xi xi
2 Baek SM. Two new cutaneous free flaps: the medial and lateral thigh flaps. Plast Reconstr Surg 1983;71(3):354-365

3 Song YG, Chen GZ, Song YL. The free thigh flap: a new free flap concept based on the septocutaneous artery. Br J Plast Surg 1984; 37(2):149-159

4 Wei FC, Jain V, Celik N, Chen HC, Chuang DC, Lin CH. Have we found an ideal soft-tissue flap? An experience with 672 anterolateral thigh flaps. Plast Reconstr Surg 2002;109(7):2219-2226, discussion 2227-2230

5 Kimura N, Satoh K. Consideration of a thin flap as an entity and clinical applications of the thin anterolateral thigh flap. Plast Reconstr Surg 1996;97(5):985-992

6 Kimura N, Saitoh M, Hasumi T, Sumiya N, Itoh Y. Clinical application and refinement of the microdissected thin groin flap transfer operation. J Plast Reconstr Aesthet Surg 2009;62(11):1510-1516

7 Gottlieb LJ, Krieger LM. From the reconstructive ladder to the reconstructive elevator. Plast Reconstr Surg 1994;93(7):1503-1504

8 Ahmadzadeh R, Bergeron L, Tang M, Morris SF. The superior and inferior gluteal artery perforator flaps. Plast Reconstr Surg 2007; 120(6):1551-1556

9 Celik N, Wei FC, Lin CH, et al. Technique and strategy in anterolateral thigh perforator flap surgery, based on an analysis of 15 complete and partial failures in 439 cases. Plast Reconstr Surg 2002;109(7):2211-2216, discussion 2217-2218

10 Hong JP, Sun SH, Ben-Nakhi M. Modified superficial circumflex iliac artery perforator flap and supermicrosurgery technique for lower extremity reconstruction: a new approach for moderatesized defects. Ann Plast Surg 2013;71(4):380-383

11 Hong JP, Koshima I. Using perforators as recipient vessels (supermicrosurgery) for free flap reconstruction of the knee region. Ann Plast Surg 2010;64(3):291-293

12 Celik N, Wei FC. Technical tips in perforator flap harvest. Clin Plast Surg 2003;30(3):469-472

13 Ross GL, Dunn R, Kirkpatrick J, et al. To thin or not to thin: the use of the anterolateral thigh flap in the reconstruction of intraoral defects. Br J Plast Surg 2003;56(4):409-413

14 Sharabi SE, Hatef DA, Koshy JC, Jain A, Cole PD, Hollier LH Jr. Is primary thinning of the anterolateral thigh flap recommended? Ann Plast Surg 2010;65(6):555-559

15 Tsai FC, Yang JY, Mardini S, Chuang SS, Wei FC. Free splitcutaneous perforator flaps procured using a three-dimensional harvest technique for the reconstruction of postburn contracture defects. Plast Reconstr Surg 2004;113(1):185-193, discussion 194-195

16 Yang WG, Chiang YC, Wei FC, Feng GM, Chen KT. Thin anterolateral thigh perforator flap using a modified perforator microdissection technique and its clinical application for foot resurfacing. Plast Reconstr Surg 2006;117(3):1004-1008

17 Alkureishi LW, Shaw-Dunn J, Ross GL. Effects of thinning the anterolateral thigh flap on the blood supply to the skin. Br J Plast Surg 2003;56(4):401-408

18 Saint-Cyr M, Wong C, Schaverien M, Mojallal A, Rohrich RJ. The perforasome theory: vascular anatomy and clinical implications. Plast Reconstr Surg 2009;124(5):1529-1544

19 Saint-Cyr M, Schaverien M, Wong C, et al. The extended anterolateral thigh flap: anatomical basis and clinical experience. Plast Reconstr Surg 2009;123(4):1245-1255

20 Hwang K, Kim DJ, Lee IJ. An anatomic comparison of the skin of five donor sites for dermal fat graft. Ann Plast Surg 2001;46(3): 327-331

21 Lee Y, Hwang K. Skin thickness of Korean adults. Surg Radiol Anat 2002;24(3-4):183-189 